\title{
Emergent Transjugular Intrahepatic Portosystemic Shunt Creation in Acute Variceal Bleeding
}

\author{
Mithil B. Pandhi, DO ${ }^{1}$ Andrew J. Kuei, MD ${ }^{1}$ Andrew J. Lipnik, MD ${ }^{1}$ Ron C. Gaba, MD, MS ${ }^{1,2}$ \\ ${ }^{1}$ Department of Radiology, University of Illinois at Chicago, Chicago, Illinois \\ 2 Division of Interventional Radiology, University of Illinois at Chicago, \\ Chicago, Illinois

\begin{abstract}
Address for correspondence Ron C. Gaba, MD, MS, Division of Interventional Radiology, Department of Radiology and Pathology, College of Medicine, University of Illinois at Chicago, 1740 West Taylor Street, MC 931, Chicago, IL 60612 (e-mail: rgaba@uic.edu).
\end{abstract}

Semin Intervent Radiol 2020;37:3-13

\begin{abstract}
Emergent transjugular intrahepatic portosystemic shunt (TIPS) creation is most commonly employed in the setting of acute variceal hemorrhage. Given a propensity for decompensation, these patients often require a multidisciplinary, multimodal approach involving prompt diagnosis, pharmacologic therapy, and endoscopic intervention. While successful in the majority of cases, failure to medically control initial

Keywords

- TIPS

- varices

- hemorrhage

- portal hypertension

- interventional radiology

bleeding can prompt interventional radiology consultation for emergent portal decompression via TIPS creation. This article discusses TIPS creation in emergent, acute variceal hemorrhage, reviewing the natural history of gastroesophageal varices, presentation and diagnosis of acute variceal hemorrhage, pharmacologic therapy, endoscopic approaches, patient selection and risk stratification for TIPS, technical considerations for TIPS creation, adjunctive embolotherapy, and the role of salvage TIPS versus early TIPS in acute variceal hemorrhage.
\end{abstract}

Emergent transjugular intrahepatic portosystemic shunt (TIPS) creation is most commonly employed in the setting of acute variceal hemorrhage ( $\mathrm{VH})$, a dreaded complication of portal hypertension in patients with liver cirrhosis. Given a propensity for decompensation, these patients often require a multidisciplinary, multimodal approach involving prompt diagnosis, pharmacologic therapy, and endoscopic intervention. ${ }^{1-4}$ While successful in the majority of cases, failure to medically control initial bleeding can prompt interventional radiology (IR) consultation for emergent portal decompression via TIPS creation. This article discusses TIPS creation in emergent, acute VH, reviewing the natural history of gastroesophageal varices (GEVs), presentation and diagnosis of acute $\mathrm{VH}$, pharmacologic therapy, endoscopic approaches, patient selection and risk stratification for TIPS, technical considerations for TIPS creation, adjunctive embolotherapy, and the role of salvage TIPS versus early TIPS in acute $\mathrm{VH}$.

\section{Gastroesophageal Varices}

Portal hypertensive hepatic venous pressure gradients (HPVGs)-defined as pressure gradients $\geq 10 \mathrm{~mm} \mathrm{Hg}$-result in GEV formation via a dynamic, multifactorial process. ${ }^{3,5}$ GEVs progress with worsening liver cirrhosis, and are categorized as "small" ( $<5 \mathrm{~mm}$ ), "medium" (based on tortuous morphology and occupation of less than one-third of the esophageal lumen), and "large" (> $5 \mathrm{~mm})^{3,6-8}$ Classically, EVs are reported in $52 \%$ of endoscopically screened patients, and increase in prevalence with disease severity, conservatively ranging from $43 \%$ in patients with Child-Pugh class A disease to $72 \%$ of patients with Child-Pugh class B/C disease. ${ }^{9}$ EVs are reported to have an annual formation incidence of 7 to $9 \%{ }^{10-12}$ and an annual progression rate of 10 to $15 \% .{ }^{13-15}$ Given these rates, approximately $90 \%$ of cirrhotic patients have EVs at 10 years. ${ }^{16}$ In contrast, GVs are less prevalent, and are reported in 5 to $33 \%$ of patients with liver cirrhosis. ${ }^{17,18}$ Today, EVs and GVs are delineated as unique anatomic entities with varying physiologic behavior. EVs exist at higher pressures than GV: 16 to $24 \mathrm{~mm} \mathrm{Hg}$ vs. 11 to $18 \mathrm{~mm} \mathrm{Hg} .^{13,14}$ While problematic EVs are historically located within the lower $5 \mathrm{~cm}$ of the distal esophagus, ${ }^{19,20} \mathrm{GVs}$ exist at locations which are demarcated by the Sarin classification system. ${ }^{17}$ In this classification scheme, gastroesophageal varices type 1 (GEV1) occur at the lesser curvature of the stomach, gastroesophageal varices type
Issue Theme Emergency IR; Guest Editors, Brian Funaki, MD and Charles E. Ray, Jr., MD, PhD, FSIR
Copyright (C) 2020 by Thieme Medical Publishers, Inc., 333 Seventh Avenue, New York, NY 10001, USA. Tel: +1(212) 760-0888.
DOI https://doi.org/ 10.1055/s-0039-3402015. ISSN $0739-9529$. 
2 (GEV2) at the greater curvature of the stomach, and isolated gastric varices type 1 (IGV1) at the fundal region. Ectopic isolated gastric varices type 2 (IGV2) are rarely encountered.

EV hemorrhage is generally thought to account for up to $70 \%$ of upper gastrointestinal (GI) bleeding in patients with liver cirrhosis, ${ }^{1,21}$ and occurs at variable rates. Small EV hemorrhage is known to occur at 5\% yearly, versus $15 \%$ annually for medium and large EV hemorrhage. ${ }^{22}$ Comparatively, GV hemorrhage occurs 50\% less frequently than EV hemorrhage, and is attributed to between 10 and $30 \%$ of all VH. ${ }^{17}$ Although less frequent, GV hemorrhage rates are reported to occur at $16 \%$ at 1 year, $36 \%$ at 3 years, and $44 \%$ at 5 years, often with high transfusion requirements due to severity of bleeding. ${ }^{17,18}$ Data show that GV hemorrhage occurs at lower portosystemic gradients (PSGs)-approximating 16 to $20 \mathrm{~mm} \mathrm{Hg}$-when compared with EV hemorrhage (21-23 $\mathrm{mm} \mathrm{Hg}$ ), with $10 \%$ of GV hemorrhage and $2 \%$ of EV hemorrhage occurring at PSGs below $12 \mathrm{~mm} \mathrm{Hg.}{ }^{23,24}$

\section{Prevention of First Variceal Hemorrhage}

Given the high prevalence of varices in liver cirrhosis, patients with this condition undergo screening endoscopy at the time of liver disease diagnosis, with follow-up surveillance every 2 to 3 years, or every 1 to 2 years if small varices are present. $3,25,26$ Additionally, endoscopy is recommended upon declaration of cirrhotic decompensation and yearly thereafter. ${ }^{3,726}$ Ideally, this strategy allows for prevention of first $\mathrm{VH}$.

Prevention of $\mathrm{VH}$ is best understood in the setting of EV. Treatment hinges on maintaining a protective HPVG $\leq 12 \mathrm{~mm}$ $\mathrm{Hg}{ }^{3,27}$ with definitive endoscopic treatment of varices. Patients with medium and large EVs, and those with decompensated cirrhosis or stigmata of bleeding-such as red wale signs-are often treated with nonselective $\beta$-blockade (using propranolol, nadolol, or carvedilol) or endoscopic variceal ligation (EVL) to potentially prevent variceal progression and hemorrhage. . $^{3,25,28,29}$ Combination therapy is usually not performed. $^{3}$

Comparatively, prevention of GV hemorrhage is less understood. A randomized controlled trial (RCT) examined the use of sclerosant glue compared with nonselective $\beta$-blockade or observation and demonstrated similar survival between $\beta$ blockade and sclerosant utilization. ${ }^{3,30}$ Prospective evaluation of variceal obliteration is yet to be adequately performed in this setting. ${ }^{31}$

\section{Diagnosis of Variceal Hemorrhage}

Patients with acute VH often present with hematemesis or melena, and variable states of hemodynamic stability. Assessment begins by obtaining a detailed history from the patient or family, complemented by thorough chart and imaging review, which will often yield a history of liver cirrhosis, portal hypertension, and/or GEVs. Baseline laboratory assessment includes a complete blood count, comprehensive metabolic panel, and a basic coagulation panel.

Differentiation between acute $\mathrm{VH}$ and other sources of upper GI bleeding in the cirrhotic patient is an important

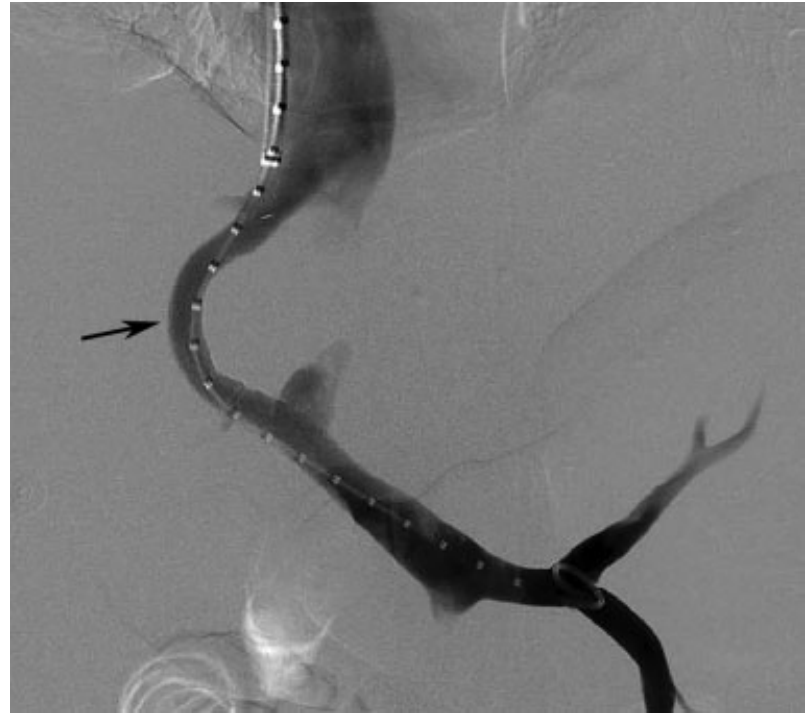

Fig. 1 TIPS in the setting of elevated MELD score. A 49-year-old woman with hepatitis $C$ virus liver cirrhosis referred to IR for emergent TIPS in the setting of massive EV bleeding and disseminated intravascular coagulation. At the time of referral, patient failed endoscopic treatment and was in critical condition with calculated MELD score of 40. TIPS created uneventfully (arrow), and laboratory and clinical findings following procedure supported bleeding cessation. Nonetheless, patient's condition did not improve, a do-not resuscitate status was assigned, and she died due to multiorgan failure 2 days post-TIPS. Though TIPS may be applied in acutely bleeding patients with high MELD scores, likelihood of survival is extremely poor. Given poor anticipated clinical outcomes, open discussion that conveys anticipated course to patient and family is critical in clinical decisionmaking process.

consideration, as this population experiences nontrivial rates of peptic ulcer disease. ${ }^{32,33}$ As the interventional approach to arterial and variceal bleeding sources differs substantially, clear delineation of the hemorrhage source is compulsory (-Fig. 1). While endoscopy serves as the primary means to diagnose bleeding sources, multiphase computed tomography angiography (CTA) may serve as an adjunctive modality to determine whether there is active arterial hemorrhage, determine a source, and provide a potential roadmap for intervention. ${ }^{34}$ Additionally, a properly performed venous phase can help identify ectopic (e.g., small bowel) varices that cannot be identified with conventional endoscopy. Intravenous proton pump inhibitors (PPIs) are often initiated in the setting of upper GI bleeding, and their use remains controversial in cirrhotic patients. ${ }^{1}$ PPIs may be discontinued upon confirmation of $\mathrm{VH}^{4}$ given an association with spontaneous bacterial peritonitis. ${ }^{35}$

\section{Pharmacotherapy, Endoscopy, and Bridge to Salvage TIPS}

Upon confirmation of acute $\mathrm{VH}$, treatment aims to achieve hemostasis, prevent early rebleeding, and minimize 6-week mortality. ${ }^{3,25,36}$ Patients experiencing acute VH demand high acuity of care, often requiring monitoring within an intensive care unit equipped with skilled nursing staff and a multidisciplinary team of critical care specialists, hepatologists, GI endoscopists, interventional radiologists (IRs), and surgeons. ${ }^{26}$ 
Initial management involves airway control, especially in the setting of hematemesis and hepatic encephalopathy (HE). Prophylactic intubation is controversial ${ }^{26}$ and has been associated with higher risk of aspiration pneumonia. ${ }^{37}$ Thus, the decision to intubate is made on a case-by-case basis.

Volume resuscitation is challenging, and conservative transfusion protocols are utilized. A mean arterial pressure goal of approximately $65 \mathrm{~mm} \mathrm{Hg}$-extrapolated from data regarding the management of septic shock and trauma ${ }^{38-41}$ -is usually pursued. ${ }^{1}$ Hypovolemia at presentation may temper VH by decreasing portal pressure, while aggressive resuscitation can aggravate $\mathrm{VH}$ or promote rebleeding by elevating portal pressure. ${ }^{4,42,43}$ A RCT demonstrated improved survival in patients with Child-Pugh class $A$ and $B$ disease, with decreased overall rebleeding in all Child-Pugh class A, B, and $C$ patients, when transfusion occurred below hemoglobin of $7 \mathrm{~g} / \mathrm{dL}$ with a posttransfusion target of 7 to $9 \mathrm{~g} / \mathrm{dL}$. ${ }^{4,44}$ Current treatment recommendations incorporate these parameters. ${ }^{3}$

Pharmacologic therapy to achieve hemostasis emphasizes splanchnic vasoconstriction in lieu of correction of coagulopathy. No benefit has been shown when factor VII is administered to correct prothrombin time. ${ }^{4,45,46}$ Moreover, no compelling data are available regarding correction of thrombocytopenia or use of desmopressin. ${ }^{3,4,47}$ Current pharmacologic options to achieve hemostasis include intravenous splanchnic vasoconstriction with octreotide, somatostatin, or terlipressin, administered for up to 3 to 5 days.,25,48 This recommendation is supported by a meta-analysis of RCTs that demonstrated improved hemostasis, decreased blood transfusions, and lower 7-day mortality with utilization of splanchnic vasoconstriction. .,49 $^{3}$

Antibiotic prophylaxis is initiated, given high risk for cirrhotic patients with upper GI bleeding to develop gramnegative bacterial infections, ${ }^{1,50-52}$ which are postulated to occur due to translocation of enteral flora. RCTs and metaanalyses exploring antibiotic prophylaxis have demonstrated decreased infection rates, rebleeding, and mortality. ${ }^{3,50,52}$ Due to prevalent quinolone resistance, $1 \mathrm{~g}$ ceftriaxone is usually administered every 24 hours for 7 days. ${ }^{3,25,53}$

Prompt endoscopy (within 12 hours) is recommended, 3,25 as patients experiencing $\mathrm{VH}$ may rapidly decompensate. Endoscopy allows for definitive treatment of bleeding varices by EVL or endoscopic injection sclerotherapy (EIS). ${ }^{26,31,54,55}$ Data show increased in-hospital mortality when endoscopy is delayed by more than 15 hours in hemodynamically stable patients, ${ }^{4,56}$ and that endoscopy within 12 hours is associated with lower rates of rebleeding and mortality. ${ }^{57}$ Timing remains debated, as others report no overall impact on mortality with delayed endoscopy in stable patients. ${ }^{56,58,59}$

While EV hemorrhage is typically treated by combination EVL and splanchnic vasoconstriction, ${ }^{3,4,25,48} \mathrm{GV}$ hemorrhage is often difficult to ameliorate by EVL secondary to anatomic considerations, and suboptimal EVL can lead to ulceration and subsequent bleeding. ${ }^{31,60}$ While a meta-analysis has shown that cyanoacrylate glue is associated with lower rebleeding rates in GVs, EVL and EIS have comparable rates of hemostasis. ${ }^{3,61}$

Despite attempts to optimize combined pharmacotherapy and endoscopic treatment, acute $\mathrm{VH}$ is refractory in approxi- mately 10 to $20 \%$ of patients, ${ }^{16,62}$ and temporizing measures must be considered prior to rapid portal decompression via TIPS creation. Today, bridging measures such as self-expanding metal stent (SEMS) deployment or 24-hour balloon tamponade (using Sengstaken-Blakemore, Linton-Nachlas, or Minnesota tubes) are utilized. Overall, utilization of balloon tamponade correlates with severity of bleeding and is successful in up to $80 \%$ of patients, although its use in $\mathrm{VH}$ is associated with increased adverse events and mortality rates of $20 \%{ }^{3,7,63,64}$ Comparatively, SEMS can be left in place for up to 7 days ${ }^{4}$ and have demonstrated both better hemostasis and less adverse events, ${ }^{3,65-67}$ although stent migration remains a worrisome complication. $^{68}$

\section{TIPS Indications}

Current indications for TIPS creation in VH are summarized by the American Association for the Study of Liver Diseases $(\mathrm{AASLD})^{3}$ :

1. Rescue therapy for acute VH which is refractory to pharmacologic and endoscopic treatment.

2. Recurrent VH despite optimization of pharmacology and endoscopic treatment.

3. Emerging indication for early (within 24-72 hours) TIPS creation in acute $\mathrm{VH}$.

TIPS is not pursued for prevention of $\mathrm{VH}$ in nonbleeding varices, prevention of rebleeding in patients who have experienced only one episode of EV hemorrhage, prevention of hemorrhage from gastric antral vascular ectasia, or portal hypertensive gastropathy. ${ }^{69,70}$

While no absolute contraindications exist, relative contraindications for TIPS creation are presented by the Society for Interventional Radiology. ${ }^{71}$ Briefly, relative contraindications include heart failure or major valvular insufficiency, elevated left or right heart pressures, rapidly progressive liver failure, severe or uncontrolled HE, uncontrolled systemic infection, sepsis, unrelieved biliary obstruction, polycystic liver disease, extensive primary or metastatic hepatic malignancy, and severe uncontrolled coagulopathy. ${ }^{71}$

\section{Patient Selection and Risk Stratification}

Patient selection is a challenging endeavor for the IR, as individuals experiencing acute $\mathrm{VH}$ often have decompensated cirrhosis. It has long been known that the markedly elevated portal pressures in decompensated cirrhosis contribute to morbidity and mortality in VH. HPVG measurements $\geq 20 \mathrm{~mm}$ $\mathrm{Hg}$ are associated with failure to achieve hemostasis and early rebleeding. ${ }^{72,73}$ Moreover, HPVGs $\geq 16 \mathrm{~mm} \mathrm{Hg}$ have also been associated with mortality ${ }^{22}$ and elevated serum bilirubin has been reported to increase risk of mortality with each $1 \mathrm{mg} / \mathrm{dL}$ increase above a threshold of $3.0 \mathrm{mg} / \mathrm{dL}^{74}$ These and other objective surrogate markers guide decision making.

Currently, the Model for End-Stage Liver Disease (MELD) score is the predominant system utilized for objective risk stratification and patient selection for TIPS creation. ${ }^{75}$ MELD utilizes serum total bilirubin, international normalized ratio 
(INR), and serum creatinine as surrogate markers to assess liver function and has classically and contemporaneously (with and without modification) demonstrated efficacy in predicting mortality. ${ }^{76-82}$ Moderately elevated short-term mortality rates are evident in patients with MELD scores exceeding 18, and markedly elevated short-term mortality rates occur above the MELD threshold of 25 ( - Fig. 2) ${ }^{83}$ Moreover, the MELD score has successfully been extrapolated in the emergent setting to predict 90-day mortality with high accuracy (area under the receiver operating characteristics: $0.842,95 \%$ confidence interval $[\mathrm{CI}]: 0.755-0.928) .{ }^{84}$ Similarly, Conejo et al examined Child-Pugh and MELD scores in patients with decompensated cirrhosis experiencing acute $\mathrm{VH}$, and reported that MELD scores exceeding 19 and Child-Pugh class $C$ disease confer high risk of death. ${ }^{85}$

Even with high predictive value, criteria such as MELD score cannot be solely utilized for patient selection. Despite poor prognosis in severely decompensated liver disease, emergent portal decompression by TIPS creation is considered a potentially lifesaving measure in refractory acute VH. Thus, patients should be selected on a case-by-case basis. Critical conversations, including a potential need for liver transplantation, are necessary between the patient (or family), referring service, and operating IR. Minor complication rates (up to $4 \%$ ) should be discussed, along with major complication rates (up to 3\%), and relevant potential risks including persistent $\mathrm{VH}$ (i.e., therapeutic failure), hepatic infarction, severe or life-altering $\mathrm{HE}$, and death. ${ }^{71}$ Additionally, if GV hemorrhage is suspected, there is potential to offer an alternative endovascular approach that obliterates bleeding GVs with or without TIPS creation. ${ }^{86}$

\section{TIPS Technical Considerations and Postprocedural Care}

Since the conceptualization of TIPS over 40 years ago, technical considerations for shunt creation have been described thoroughly and revisited in detail. ${ }^{87-89}$ TIPS creation commonly involves the formation of a tract between the right hepatic vein and right portal vein. This is most commonly achieved by selecting the right hepatic vein and utilizing wedged hepatic venography to identify the right portal vein. A needle is fluoroscopically targeted toward a right portal vein branch. The PSG may be calculated once successful portal venous access has been achieved. Contemporary TIPS are generated by deployment of a stent graft (Viatorr; W.L. Gore, Flagstaff, AZ) for shunt creation. Bare metal stents are rarely used in modern TIPS procedures, given reduced overall patency and association with increased rebleeding rates and decreased survival ${ }^{87,90-94}$; nonetheless, there may be specific clinical scenarios that merit consideration of bare metal stent TIPS creation ( - Fig. 3). After stent-graft deployment, the shunt tract is dilated incrementally with an 8- to 10 -mm balloon to a target PSG $\leq 12 \mathrm{~mm} \mathrm{Hg.}{ }^{89}$ Additional splenic and portal venography can be performed to detect varices, which may serve as targets for embolization, most commonly the left gastric (coronary) vein, posterior gastric vein, and short gastric vein. ${ }^{89,95}$ Moreover, if GVs are present, the operator may choose to obliterate varices at the time of TIPS creation or pursue staged obliteration at a later
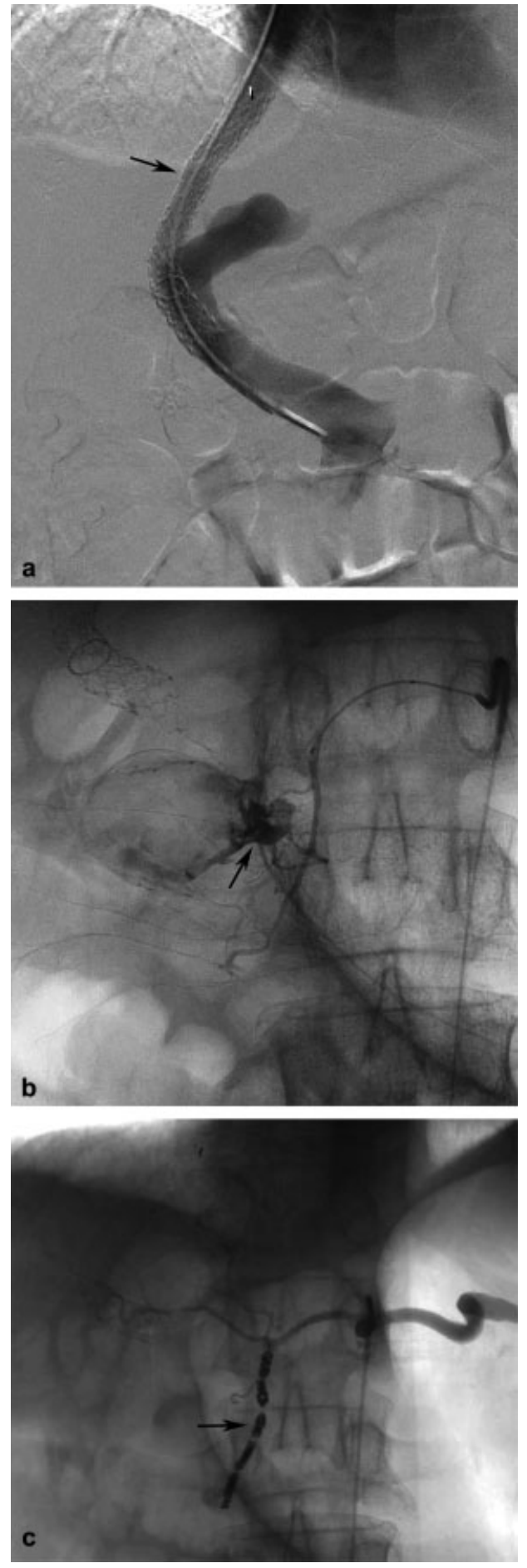

Fig. 2 TIPS for presumed variceal bleeding with subsequent discovery of arterial bleeding source. A 73-year-old man with alcoholic cirrhosis and acute $\mathrm{VH}$ transferred from outside hospital for possible TIPS creation. Upper endoscopy performed 1 week prior revealed bleeding EVs. Upon transfer, recurrent EV bleeding presumed, though active bleeding source not established via repeat endoscopy. While TIPS was successfully created (a; arrow), patient continued to have hematochezia and decreasing hemoglobin levels. Repeat upper endoscopy revealed bleeding duodenal ulcer. Arteriography confirmed active hemorrhage from gastroduodenal artery (b; arrow), and bleeding cessation achieved after coil embolization (c; arrow). Appropriate preprocedural diagnosis and workup is critical in establishing source of upper $\mathrm{Gl}$ bleeding prior to therapy prescription, to recommend most suitable management approach. 

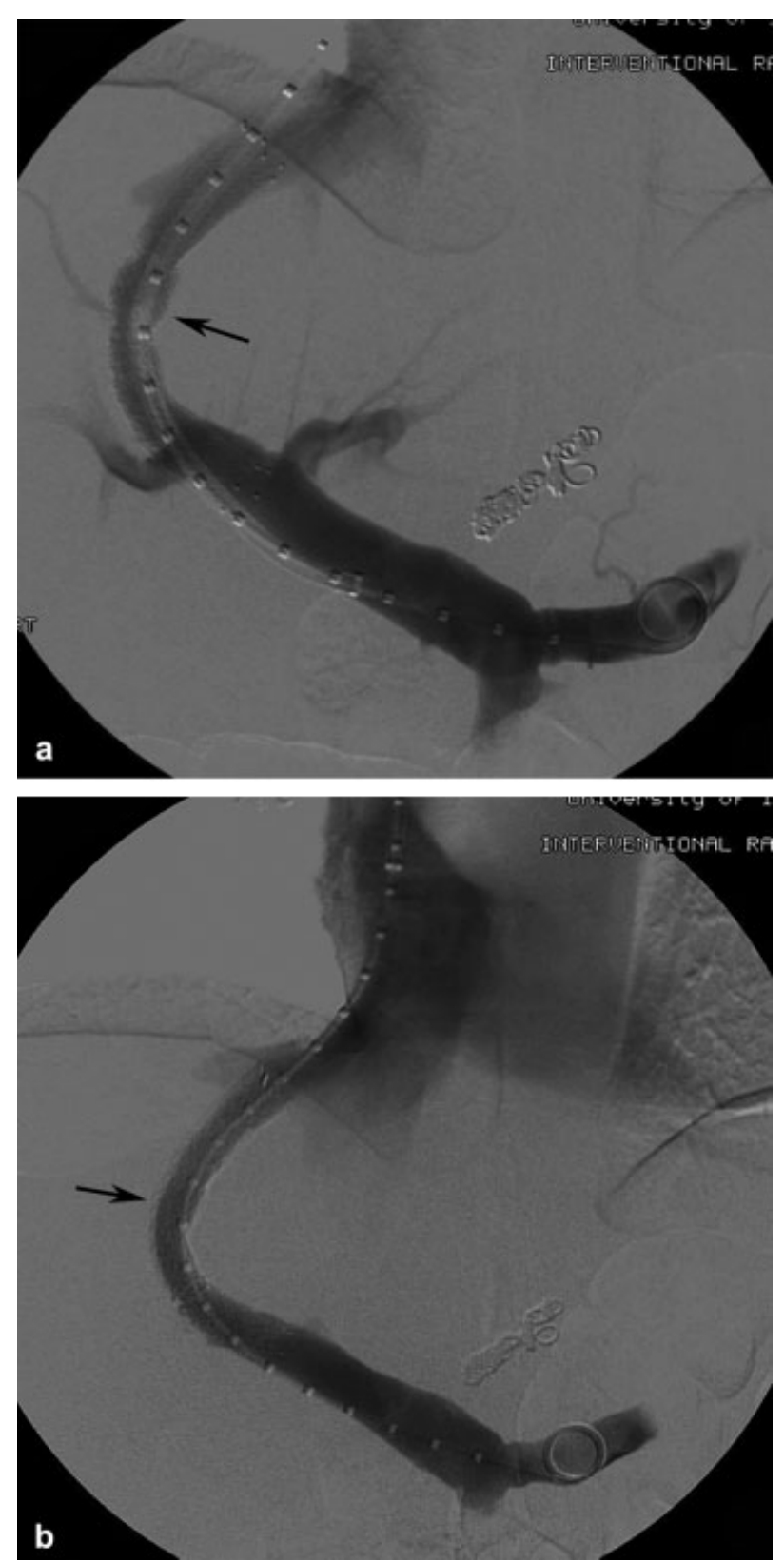

Fig. 3 Bare metal stent TIPS in the setting of bacteremia. A 63-year-old man transferred for management of actively bleeding EVs after unsuccessful banding. Patient presented with fever, leukocytosis, and computed tomography findings of colitis. There was clinical concern for bacteremia, though culture results were pending. TIPS was created using bare metal stent (a; arrow) to avoid potential risk of stent-graft colonization, with plan to revise stent-graft TIPS when blood cultures confirmed to be sterile. After TIPS, bleeding stopped, and patient returned 5 days later for successful shunt relining using PTFE-covered stent graft (b; arrow). Infection of TIPS stent grafts-termed endotipsitis-is an infrequent but serious complication associated with high mortality rates. As such, bacteremia or uncontrolled sepsis is considered a relative contraindication for TIPS creation. In such scenarios, bare metal stent may offer lower risk of colonization, given absence of covering graft material.

date. ${ }^{86}$ Importantly, balloon tamponade devices need to be deflated after shunt creation to ensure variceal decompression and to allow catheter access to varices if embolization or obliteration is to be pursued. Another important consideration when treating acute $\mathrm{VH}$ is preprocedural correction of coagul- opathy, ${ }^{96}$ which is often not pursued in the cirrhotic patient prior to salvage therapy.

In the post-TIPS period, the acute VH patient is generally monitored in the intensive care unit to monitor for hemodynamic stability, resolution of hemorrhage, TIPS-related adverse events, and neurological signs of HE. ${ }^{86}$ Alongside monitoring nasogastric tube output, serial hemoglobin and hematocrit values may be obtained to monitor cessation of VH. A follow-up liver panel and coagulation panel are also obtained the day after the procedure to assess hepatic function. TIPS patency may be monitored with serial Doppler ultrasound at 1, 3, and 6 months postprocedure.

\section{TIPS Creation for Initial Control of VH}

TIPS creation is nearly always technically feasible and is a bona fide treatment for initial control of acute $\mathrm{VH}^{75,97}$ as historical datasets report rates of hemostasis between 89 and 100\%, ${ }^{98-109}$ largely in EV hemorrhage. However, historical cohorts have demonstrated variable rebleeding rates, ${ }^{97}$ which may be inflated due to early widespread utilization of uncovered bare metal stents prior to the advent of the bile impermeable polytetrafluoroethylene (PTFE) stent grafts, which prevent bilemediated thrombosis and TIPS dysfunction. ${ }^{110}$ For instance, a recent retrospective cohort demonstrated significantly lower rebleeding rates ( 14 vs. $37 \%$, odds ratio $[\mathrm{OR}]=0.259 ; p<0.001$ ) and need for shunt revision when comparing PTFE TIPS stent grafts with bare metal stents. ${ }^{93}$

Historically, studies have reported variable short-term mortality rates for salvage TIPS, ranging between 19 and $60 \%{ }^{97}$ Tenuous outcomes continue to be reported for salvage TIPS in hemodynamically unstable patients. Active hemorrhage at the time of TIPS creation has been associated with a 2.9-fold greater odds of death. ${ }^{74,111}$ A recent retrospective analysis reported approximately $34 \%$ mortality at 30 days in patients with hemorrhagic shock undergoing salvage TIPS, in addition to overall unfavorable clinical outcome. ${ }^{112}$

While studies examining TIPS in acute VH are variable due to inclusion criteria and methodology, recent studies demonstrate similar outcomes for rebleeding and overall survival. Gaba et al reported 1-month rebleeding rates less than 10\% with rebleeding rates of $22 \%$ at 1 year and nearly $30 \%$ at 2 years, and survival rates of 80,69 , and $65 \%$ at 30 days, 1 -year, and 2 years, respectively. ${ }^{113} \mathrm{Zhu}$ et al recently reported similar findings, including rebleeding rates of 11,17 , and $20 \%$ at 6 weeks, 1 year, and 2 years, respectively. ${ }^{114}$ Transplant-free survival was reported to be 88,82 , and $74 \%$ at 6 weeks, 1 year, and 2 years, respectively. ${ }^{114}$ Longer term survival data remain sparse. Notably, Sanyal et al reported $46 \%$ survival at a median follow-up period of 920 days after salvage TIPS, while Azoulay et al reported 1-year survival of 51 and $40 \%$ at 3 and 5 years $^{98,105}$; however, these datasets preceded the era of PTFE-covered stent grafts.

Additionally, comparison between emergent TIPS and creation of a surgical portosystemic shunt (SPSS) remains debated. A recent systematic review demonstrated low confidence when assessing RCTs that demonstrated higher rates of all-cause mortality (RR: $0.61,95 \% \mathrm{CI}: 0.42-0.90$ ), 
rebleeding (RR: 0.19, 95\% CI: 0.08-0.49), reintervention (RR: 0.13, 95\% CI: 0.06-0.28), and shunt occlusion (RR: 0.14, 95\% CI: $0.040-0.51)$ at 5 years with TIPS versus SPSS. Given the high numbers of bare metal stents utilized in available datasets, ${ }^{115-118}$ prospective data exclusively utilizing PTFEcovered TIPS are necessary.

\section{TIPS Creation for GV Hemorrhage}

TIPS for GV hemorrhage requires more data. TIPS has been reported to achieve bleeding control in $90 \%$ of bleeding $\mathrm{GVs}^{107,119}$ and, in a RCT, demonstrated significantly lower rebleeding from GV hemorrhage when compared with endoscopic glue obliteration at a median follow-up of 33 months. ${ }^{120}$ However, recent data suggest that TIPS may not be the optimal modality for addressing GV hemorrhage, which bleed at lower pressures with recent data demonstrating a rebleeding rate of 27 and $15 \%$ mortality at 90 days. ${ }^{121}$ Therefore, patients presenting with GV hemorrhage may be considered for concurrent obliteration in addition to TIPS. ${ }^{86}$

\section{Early TIPS Creation for Acute VH}

Early nonsalvage TIPS creation (usually within 72 hours) is an emerging indication in VH with promising results. Early TIPS supplements initiated pharmacologic and endoscopic interventions, and is theorized to mitigate background elevated portal pressure and prevent rebleeding. ${ }^{122}$ Initially, a RCT with 116 consecutive patients utilized uncovered stents in early VH (24hours), and demonstrated that patients with HPVG $\geq 20 \mathrm{~mm} \mathrm{Hg}$ had significantly less treatment failure (12 vs. 50\%) and transfusion requirements when compared with those not undergoing TIPS. ${ }^{123}$ This study also reported significantly lower in-hospital and 11-year mortality (11 and $31 \%$, respectively). ${ }^{123}$ Subsequently, a RCT of patients with Child-Pugh B/C VH showed improved rates of bleeding control ( 97 vs. 50\%), 6-week survival ( 97 vs. $67 \%$ ), and 1 -year actuarial survival ( 86 vs. $61 \%$ ) with a PTFE-covered TIPS versus pharmacologic and endoscopic therapy. ${ }^{124} \mathrm{~A}$ subsequent retrospective review comparing TIPS versus combination pharmacotherapy and endoscopy in the same center demonstrated similar findings, as the TIPS group experienced significantly lower rates of failure to control bleeding or rebleeding (93 vs. $50 \%$ ) and improved 1-year actuarial survival (86 vs. $70 \%$ ). ${ }^{125}$ Another prospective study aimed at external validation of initial results, and demonstrated significantly lower 1-year probability for rebleeding with early TIPS creation in a similar patient cohort (97 vs. 51\%; $p<0.001$ ), but reported no difference in actuarial survival. ${ }^{126}$

Recent data are also available. A multicenter observational study comparing early TIPS and combination pharmacotherapy and endoscopy in 631 patients demonstrated significantly improved survival at 1 year when comparing early TIPS to combination pharmacotherapy and endoscopy in patients with Child-Pugh $\mathrm{C}$ disease along with reduced treatment failure (which incorporated rebleeding). ${ }^{60}$ Additionally, a recent RCT examining early covered TIPS with pharmacologic therapy and endoscopy examined transplant-free survival as an endpoint and demonstrated significantly improved transplant-free survival at 6 weeks and 1 year in patients who received early TIPS. ${ }^{127}$

Several meta-analyses have also been performed. An analysis from 2015 evaluated randomized and nonrandomized data in early TIPS creation, reporting fewer deaths (OR: 0.38, 95\% $\mathrm{CI}=0.17-0.83 ; p=0.02$ ) and lower rates of rebleeding within 1 year in patients with Child-Pugh B (OR: 0.08, 95\% CI: $0.04-0.17 ; p<0.001)$ and Child-Pugh C (OR $=0.05,95 \%$ $\mathrm{CI}=0.02-0.15 ; p<0.001)$ disease. ${ }^{128}$ A subsequent meta-analysis echoed these findings, reporting significantly decreased inpatient mortality $(R R=0.87)$ and rebleeding $(R R=0.56) .{ }^{129}$ Notably, early TIPS did not demonstrate increased HE in these studies, which is an important consideration for patients, families, consulting physicians, and operators.

Additionally, a 10-year retrospective inpatient dataset of 142,539 patients with decompensated cirrhosis and EV hemorrhage demonstrated significantly decreased rebleeding $(R R=0.56)$ and inpatient mortality $(R R=0.87)$ with early TIPS when compared with no TIPS. ${ }^{130}$ Significantly less inpatient rebleeding, death, and cost were observed as well. ${ }^{130}$ Additional analysis compared early TIPS versus salvage TIPS, yielding significantly less inpatient rebleeding, death, length of stay, and cost for the early TIPS group. ${ }^{130}$ These latter results may reflect the dire clinical circumstances encountered when salvage TIPS is considered.

\section{TIPS Creation with Adjunctive Embolotherapy}

Adjunctive embolotherapy of varices is a protective measure used alongside TIPS creation, and is theorized to not only result in variceal occlusion, but may also serve to maintain TIPS patency by eliminating competing variceal shunts which could contribute to TIPS dysfunction and promote subsequent GEV formation and $\mathrm{VH}^{97,131,132}$ The embolization procedure may be staged prior to or following TIPS creation. ${ }^{86}$ Post-TIPS variceal embolization allows the operator to view optimized filling of the newly created TIPS and its impact on variceal filling. Alternatively, preprocedural embolotherapy has the benefit of improved variceal filling to guide targeting. Moreover, embolotherapy performed before TIPS creation avoids an avenue for embolic agent migration and nontarget embolization.

Although controversial, adjunctive embolotherapy has been utilized in up to $48 \%$ of patients ${ }^{97,131}$ and has largely favorable results considering prospective and retrospective data. ${ }^{86}$ Prospective studies have shown significant reductions in rebleeding rates with adjunctive embolotherapy when compared with TIPS alone. ${ }^{132-134}$ Retrospective data are mixed, with a majority of studies with significant reductions in rates of rebleeding when adjunctive embolotherapy is utilized. ${ }^{135,136}$ Other retrospective data demonstrate lower, although nonsignificant, rates of rebleeding between combined treatment and TIPS alone. ${ }^{8,137,138}$ Moreover, a meta-analysis comparing combined TIPS and adjunctive embolotherapy versus TIPS alone reported that combined therapy significantly reduces rebleeding and improves TIPS patency at 6 months. ${ }^{139}$ A recent 
retrospective study exploring adjunctive embolotherapy reported nearly $6 \%$ rebleeding rate at a median follow-up time of 26 months with approximately 1 and 3\% rebleeding rates at 1 and 2 years. ${ }^{140}$

\section{Conclusion}

Patients with decompensated liver cirrhosis and acute VH require multifaceted, multidisciplinary treatment. Emergent TIPS creation successfully ameliorates acute VH when standard medical and endoscopic management are unsuccessful, and recent data demonstrate a promising role for early, nonsalvage TIPS creation in acute VH. Patient selection and risk stratification for this potentially life-saving procedure remains challenging and continues to be determined on a case-by-case basis. Decision making can be aided by objective measures such as MELD score along with anatomic considerations such as the presence of bleeding GVs which could benefit from obliteration or combined TIPS approaches.

\section{References}

1 Mallet M, Rudler M, Thabut D. Variceal bleeding in cirrhotic patients. Gastroenterol Rep (Oxf) 2017;5(03):185-192

2 Nadim MK, Durand F, Kellum JA, et al. Management of the critically ill patient with cirrhosis: a multidisciplinary perspective. J Hepatol 2016;64(03):717-735

3 Garcia-Tsao G, Abraldes JG, Berzigotti A, Bosch J. Portal hypertensive bleeding in cirrhosis: risk stratification, diagnosis, and management: 2016 practice guidance by the American Association for the study of liver diseases. Hepatology 2017;65(01):310-335

4 Zanetto A, Garcia-Tsao G. Management of acute variceal hemorrhage. F1000 Res 2019;8:8

5 Groszmann RJ, Garcia-Tsao G, Bosch J, et al; Portal Hypertension Collaborative Group. Beta-blockers to prevent gastroesophageal varices in patients with cirrhosis. N Engl J Med 2005;353(21): 2254-2261

6 de Franchis R, Pascal JP, Ancona E, et al. Definitions, methodology and therapeutic strategies in portal hypertension. A Consensus Development Workshop, Baveno, Lake Maggiore, Italy, April 5 and 6, 1990. J Hepatol 1992;15(1-2):256-261

7 Garcia-Tsao G, Sanyal AJ, Grace ND, Carey W; Practice Guidelines Committee of the American Association for the Study of Liver Diseases; Practice Parameters Committee of the American College of Gastroenterology. Prevention and management of gastroesophageal varices and variceal hemorrhage in cirrhosis. Hepatology 2007;46(03):922-938

8 Abby Philips C, Sahney A. Oesophageal and gastric varices: historical aspects, classification and grading: everything in one place. Gastroenterol Rep (Oxf) 2016;4(03):186-195

9 Kovalak M, Lake J, Mattek N, Eisen G, Lieberman D, Zaman A. Endoscopic screening for varices in cirrhotic patients: data from a national endoscopic database. Gastrointest Endosc 2007;65 (01):82-88

10 Christensen E, Fauerholdt L, Schlichting P, Juhl E, Poulsen H, Tygstrup N. Aspects of the natural history of gastrointestinal bleeding in cirrhosis and the effect of prednisone. Gastroenterology 1981;81(05):944-952

11 Merli M, Nicolini G, Angeloni S, et al. Incidence and natural history of small esophageal varices in cirrhotic patients. J Hepatol 2003;38(03):266-272

12 Gulamhusein AF, Kamath PS. The epidemiology and pathogenesis of gastrointestinal varices. Tech Gastrointest Endosc 2017;19 (02):62-68
13 Watanabe K, Kimura K, Matsutani S, Ohto M, Okuda K. Portal hemodynamics in patients with gastric varices. A study in 230 patients with esophageal and/or gastric varices using portal vein catheterization. Gastroenterology 1988;95(02):434-440

14 Chao Y, Lin HC, Lee FY, et al. Hepatic hemodynamic features in patients with esophageal or gastric varices. J Hepatol 1993;19 (01):85-89

15 Merkel C, Marin R, Angeli P, et al; Gruppo Triveneto per l'Ipertensione Portale. A placebo-controlled clinical trial of nadolol in the prophylaxis of growth of small esophageal varices in cirrhosis. Gastroenterology 2004;127(02):476-484

16 D'Amico G, Pagliaro L, Bosch J. The treatment of portal hypertension: a meta-analytic review. Hepatology 1995;22(01):332-354

17 Sarin SK, Lahoti D, Saxena SP, Murthy NS, Makwana UK. Prevalence, classification and natural history of gastric varices: a longterm follow-up study in 568 portal hypertension patients. Hepatology 1992;16(06):1343-1349

$18 \mathrm{Kim} \mathrm{T}$, Shijo H, Kokawa H, et al. Risk factors for hemorrhage from gastric fundal varices. Hepatology 1997;25(02):307-312

19 Dagradi AE. The natural history of esophageal varices in patients with alcoholic liver cirrhosis. An endoscopic and clinical study. Am J Gastroenterol 1972;57(06):520-540

20 Barbu LA, Mărgăritescu ND, Şurlin MV. Diagnosis and treatment algorithms of acute variceal bleeding. Curr Health Sci J 2017;43 (03):191-200

21 Rudler M, Rousseau G, Benosman H, et al. Peptic ulcer bleeding in patients with or without cirrhosis: different diseases but the same prognosis? Aliment Pharmacol Ther 2012;36(02):166-172

22 Merkel C, Zoli M, Siringo S, et al. Prognostic indicators of risk for first variceal bleeding in cirrhosis: a multicenter study in 711 patients to validate and improve the North Italian Endoscopic Club (NIEC) index. Am J Gastroenterol 2000;95(10):2915-2920

23 Tripathi D, Therapondos G, Jackson E, Redhead DN, Hayes PC. The role of the transjugular intrahepatic portosystemic stent shunt (TIPSS) in the management of bleeding gastric varices: clinical and haemodynamic correlations. Gut 2002;51(02):270-274

24 Morrison JD, Mendoza-Elias N, Lipnik AJ, et al. Gastric varices bleed at lower portosystemic pressure gradients than esophageal varices. J Vasc Interv Radiol 2018;29(05):636-641

25 de Franchis R; Baveno VI Faculty. Expanding consensus in portal hypertension: report of the Baveno VI Consensus Workshop: stratifying risk and individualizing care for portal hypertension. J Hepatol 2015;63(03):743-752

26 Tayyem O, Bilal M, Samuel R, Merwat SK. Evaluation and management of variceal bleeding. Dis Mon 2018;64(07): 312-320

27 Bosch J, García-Pagán JC. Prevention of variceal rebleeding. Lancet 2003;361(9361):952-954

28 D’Amico G, Pagliaro L, Bosch J. Pharmacological treatment of portal hypertension: an evidence-based approach. Semin Liver Dis 1999;19(04):475-505

29 Gluud LL, Krag A. Banding ligation versus beta-blockers for primary prevention in oesophageal varices in adults. Cochrane Database Syst Rev 2012;(08):CD004544

30 Mishra SR, Chander Sharma B, Kumar A, Sarin SK. Endoscopic cyanoacrylate injection versus beta-blocker for secondary prophylaxis of gastric variceal bleed: a randomised controlled trial. Gut 2010;59(06):729-735

31 Seo YS. Prevention and management of gastroesophageal varices. Clin Mol Hepatol 2018;24(01):20-42

32 Tandon P, Bishay K, Fisher S, et al. Comparison of clinical outcomes between variceal and non-variceal gastrointestinal bleeding in patients with cirrhosis. J Gastroenterol Hepatol 2018;33 (10):1773-1779

33 Hsu YC, Lin JT, Chen TT, Wu MS, Wu CY. Long-term risk of recurrent peptic ulcer bleeding in patients with liver cirrhosis: a 10-year nationwide cohort study. Hepatology 2012;56(02):698-705 
34 Storace M, Martin JG, Shah J, Bercu Z. CTA as an adjuvant tool for acute intra-abdominal or gastrointestinal bleeding. Tech Vasc Interv Radiol 2017;20(04):248-257

35 Trikudanathan G, Israel J, Cappa J, O'Sullivan DM. Association between proton pump inhibitors and spontaneous bacterial peritonitis in cirrhotic patients - a systematic review and meta-analysis. Int J Clin Pract 2011;65(06):674-678

36 de Franchis R, Dell'Era A. Invasive and noninvasive methods to diagnose portal hypertension and esophageal varices. Clin Liver Dis 2014;18(02):293-302

37 Koch DG, Arguedas MR, Fallon MB. Risk of aspiration pneumonia in suspected variceal hemorrhage: the value of prophylactic endotracheal intubation prior to endoscopy. Dig Dis Sci 2007; 52(09):2225-2228

38 Dellinger RP, Levy MM, Carlet JM, et al; International Surviving Sepsis Campaign Guidelines Committee; American Association of Critical-Care Nurses; American College of Chest Physicians; American College of Emergency Physicians; Canadian Critical Care Society; European Society of Clinical Microbiology and Infectious Diseases; European Society of Intensive Care Medicine; European Respiratory Society; International Sepsis Forum; Japanese Association for Acute Medicine; Japanese Society of Intensive Care Medicine; Society of Critical Care Medicine; Society of Hospital Medicine; Surgical Infection Society; World Federation of Societies of Intensive and Critical Care Medicine. Surviving Sepsis Campaign: international guidelines for management of severe sepsis and septic shock: 2008. Crit Care Med 2008;36(01):296-327

39 Dellinger RP, Levy MM, Rhodes A, et al; Surviving Sepsis Campaign Guidelines Committee including The Pediatric Subgroup. Surviving Sepsis Campaign: international guidelines for management of severe sepsis and septic shock, 2012. Intensive Care Med 2013;39(02):165-228

40 Rossaint R, Bouillon B, Cerny V, et al; Task Force for Advanced Bleeding Care in Trauma. Management of bleeding following major trauma: an updated European guideline. Crit Care 2010; 14(02):R52

41 Rossaint R, Bouillon B, Cerny V, et al. The European guideline on management of major bleeding and coagulopathy following trauma: fourth edition. Crit Care 2016;20:100

42 Kravetz D, Sikuler E, Groszmann RJ. Splanchnic and systemic hemodynamics in portal hypertensive rats during hemorrhage and blood volume restitution. Gastroenterology 1986;90(5, Pt 1):1232-1240

43 Kravetz D, Bosch J, Arderiu M, Pilar Pizcueta M, Rodés J. Hemodynamic effects of blood volume restitution following a hemorrhage in rats with portal hypertension due to cirrhosis of the liver: influence of the extent of portal-systemic shunting. Hepatology 1989;9(06):808-814

44 Villanueva C, Colomo A, Bosch A, et al. Transfusion strategies for acute upper gastrointestinal bleeding. N Engl J Med 2013;368 (01):11-21

45 Bosch J, Thabut D, Bendtsen F, et al; European Study Group on rFVIIa in UGI Haemorrhage. Recombinant factor VIIa for upper gastrointestinal bleeding in patients with cirrhosis: a randomized, double-blind trial. Gastroenterology 2004;127(04):1123-1130

46 Bosch J, Thabut D, Albillos A, et al; International Study Group on rFVIIa in UGI Hemorrhage. Recombinant factor VIIa for variceal bleeding in patients with advanced cirrhosis: a randomized, controlled trial. Hepatology 2008;47(05):1604-1614

47 de Franchis R, Arcidiacono PG, Carpinelli L, et al. Randomized controlled trial of desmopressin plus terlipressin vs. terlipressin alone for the treatment of acute variceal hemorrhage in cirrhotic patients: a multicenter, double-blind study. New Italian Endoscopic Club. Hepatology 1993;18(05):1102-1107

48 European Association for the Study of the Liver. Electronic address: easloffice@easloffice.eu; European Association for the Study of the Liver. EASL Clinical Practice Guidelines for the management of patients with decompensated cirrhosis. J Hepatol 2018;69(02):406-460

49 Wells M, Chande N, Adams P, et al. Meta-analysis: vasoactive medications for the management of acute variceal bleeds. Aliment Pharmacol Ther 2012;35(11):1267-1278

50 Bernard B, Grangé JD, Khac EN, Amiot X, Opolon P, Poynard T. Antibiotic prophylaxis for the prevention of bacterial infections in cirrhotic patients with gastrointestinal bleeding: a metaanalysis. Hepatology 1999;29(06):1655-1661

51 Bernard B, Cadranel JF, Valla D, Escolano S, Jarlier V, Opolon P. Prognostic significance of bacterial infection in bleeding cirrhotic patients: a prospective study. Gastroenterology 1995;108 (06):1828-1834

52 Chavez-Tapia NC, Barrientos-Gutierrez T, Tellez-Avila F, et al. Meta-analysis: antibiotic prophylaxis for cirrhotic patients with upper gastrointestinal bleeding - an updated Cochrane review. Aliment Pharmacol Ther 2011;34(05):509-518

53 Fernández J, Ruiz del Arbol L, Gómez C, et al. Norfloxacin vs ceftriaxone in the prophylaxis of infections in patients with advanced cirrhosis and hemorrhage. Gastroenterology 2006;131 (04):1049-1056, quiz 1285

54 Villanueva C, Piqueras M, Aracil C, et al. A randomized controlled trial comparing ligation and sclerotherapy as emergency endoscopic treatment added to somatostatin in acute variceal bleeding. J Hepatol 2006;45(04):560-567

55 Dai C, Liu WX, Jiang M, Sun MJ. Endoscopic variceal ligation compared with endoscopic injection sclerotherapy for treatment of esophageal variceal hemorrhage: a meta-analysis. World J Gastroenterol 2015;21(08):2534-2541

56 Hsu YC, Chung CS, Tseng CH, et al. Delayed endoscopy as a risk factor for in-hospital mortality in cirrhotic patients with acute variceal hemorrhage. J Gastroenterol Hepatol 2009;24(07):1294-1299

57 Chen PH, Chen WC, Hou MC, et al. Delayed endoscopy increases re-bleeding and mortality in patients with hematemesis and active esophageal variceal bleeding: a cohort study. J Hepatol 2012;57(06):1207-1213

58 Cheung J, Soo I, Bastiampillai R, Zhu Q Ma M. Urgent vs. nonurgent endoscopy in stable acute variceal bleeding. Am J Gastroenterol 2009;104(05):1125-1129

59 Yoo JJ, Chang Y, Cho EJ, et al. Timing of upper gastrointestinal endoscopy does not influence short-term outcomes in patients with acute variceal bleeding. World J Gastroenterol 2018;24(44): 5025-5033

60 Hernández-Gea V, Procopet B, Giráldez Á, et al; International Variceal Bleeding Observational Study Group and Baveno Cooperation. Preemptive-TIPS improves outcome in high-risk variceal bleeding: an observational study. Hepatology 2019;69(01):282-293

61 Ríos Castellanos E, Seron P, Gisbert JP, Bonfill Cosp X. Endoscopic injection of cyanoacrylate glue versus other endoscopic procedures for acute bleeding gastric varices in people with portal hypertension. Cochrane Database Syst Rev 2015;(05):CD010180

62 Loffroy R, Estivalet L, Cherblanc V, et al. Transjugular intrahepatic portosystemic shunt for the management of acute variceal hemorrhage. World J Gastroenterol 2013;19(37):6131-6143

63 Tapper EB, Beste L, Curry M, Bonder A, Waljee A, Saini S. Suboptimal implementation of evidence-based therapy for acute variceal hemorrhage: a systematic review of observational studies. Clin Gastroenterol Hepatol 2017;15(09):1373-1381.e7

64 Nadler J, Stankovic N, Uber A, et al. Outcomes in variceal hemorrhage following the use of a balloon tamponade device. Am J Emerg Med 2017;35(10):1500-1502

65 Shao XD, Qi XS, Guo XZ. Esophageal stent for refractory variceal bleeding: a systemic review and meta-analysis. BioMed Res Int 2016;2016:4054513

66 Escorsell À, Pavel O, Cárdenas A, et al; Variceal Bleeding Study Group. Esophageal balloon tamponade versus esophageal stent in controlling acute refractory variceal bleeding: a multicenter 
randomized, controlled trial. Hepatology 2016;63(06): 1957-1967

67 Marot A, Trépo E, Doerig C, Moreno C, Moradpour D, Deltenre P. Systematic review with meta-analysis: self-expanding metal stents in patients with cirrhosis and severe or refractory oesophageal variceal bleeding. Aliment Pharmacol Ther 2015; 42(11-12):1250-1260

68 Changela K, Ona MA, Anand S, Duddempudi S. Self-expanding metal stent (SEMS): an innovative rescue therapy for refractory acute variceal bleeding. Endosc Int Open 2014;2(04):E244-E251

69 Boyer TD, Haskal ZJ. American Association for the Study of Liver Diseases Practice Guidelines: the role of transjugular intrahepatic portosystemic shunt creation in the management of portal hypertension. J Vasc Interv Radiol 2005;16(05):615-629

70 Boyer TD, Haskal ZJ; American Association for the Study of Liver Diseases. The role of transjugular intrahepatic portosystemic shunt (TIPS) in the management of portal hypertension: update 2009. Hepatology 2010;51(01):306

71 Dariushnia SR, Haskal ZJ, Midia M, et al; Society of Interventional Radiology Standards of Practice Committee. Quality improvement guidelines for transjugular intrahepatic portosystemic shunts. J Vasc Interv Radiol 2016;27(01):1-7

72 Moitinho E, Escorsell A, Bandi JC, et al. Prognostic value of early measurements of portal pressure in acute variceal bleeding. Gastroenterology 1999;117(03):626-631

73 Abraldes JG, Villanueva C, Bañares R, et al; Spanish Cooperative Group for Portal Hypertension and Variceal Bleeding. Hepatic venous pressure gradient and prognosis in patients with acute variceal bleeding treated with pharmacologic and endoscopic therapy. J Hepatol 2008;48(02):229-236

74 Rajan DK, Haskal ZJ, Clark TW. Serum bilirubin and early mortality after transjugular intrahepatic portosystemic shunts: results of a multivariate analysis. J Vasc Interv Radiol 2002;13 (2, Pt 1):155-161

75 Parvinian A, Gaba RC. Outcomes of TIPS for treatment of gastroesophageal variceal hemorrhage. Semin Intervent Radiol 2014; 31(03):252-257

76 Kamath PS, Wiesner RH, Malinchoc M, et al. A model to predict survival in patients with end-stage liver disease. Hepatology 2001;33(02):464-470

77 Salerno F, Merli M, Cazzaniga M, et al. MELD score is better than Child-Pugh score in predicting 3-month survival of patients undergoing transjugular intrahepatic portosystemic shunt. J Hepatol 2002;36(04):494-500

78 Ferral H, Gamboa P, Postoak DW, et al. Survival after elective transjugular intrahepatic portosystemic shunt creation: prediction with model for end-stage liver disease score. Radiology 2004;231(01):231-236

79 Schepke M, Roth F, Fimmers R, et al. Comparison of MELD, ChildPugh, and Emory model for the prediction of survival in patients undergoing transjugular intrahepatic portosystemic shunting. Am J Gastroenterol 2003;98(05):1167-1174

80 Rudler M, Bureau C, Carbonell N, et al; French Club for the Study of Portal Hypertension (CFEHTP). Recalibrated MELD and hepatic encephalopathy are prognostic factors in cirrhotic patients with acute variceal bleeding. Liver Int 2018;38(03):469-476

81 Allegretti AS, Frenk NE, Li DK, et al. Evaluation of model performance to predict survival after transjugular intrahepatic portosystemic shunt placement. PLoS One 2019;14(05):e0217442

82 Maimone S, Saffioti F, Filomia R, et al. Predictors of re-bleeding and mortality among patients with refractory variceal bleeding undergoing salvage transjugular intrahepatic portosystemic shunt (TIPS). Dig Dis Sci 2019;64(05):1335-1345

83 Gaba RC, Couture PM, Bui JT, et al. Prognostic capability of different liver disease scoring systems for prediction of early mortality after transjugular intrahepatic portosystemic shunt creation. J Vasc Interv Radiol 2013;24(03):411-420, 420.e1-420. e4, quiz 421
84 Casadaban LC, Parvinian A, Zivin SP, et al. MELD score for prediction of survival after emergent TIPS for acute variceal hemorrhage: derivation and validation in a 101-patient cohort. Ann Hepatol 2015;14(03):380-388

85 Conejo I, Guardascione MA, Tandon P, et al. Multicenter external validation of risk stratification criteria for patients with variceal bleeding. Clin Gastroenterol Hepatol 2018;16(01):132-139.e8

86 Lipnik AJ, Pandhi MB, Khabbaz RC, Gaba RC. Endovascular treatment for variceal hemorrhage: TIPS, BRTO, and combined approaches. Semin Intervent Radiol 2018;35(03):169-184

87 Kalva SP, Salazar GM, Walker TG. Transjugular intrahepatic portosystemic shunt for acute variceal hemorrhage. Tech Vasc Interv Radiol 2009;12(02):92-101

88 Gaba RC, Khiatani VL, Knuttinen MG, et al. Comprehensive review of TIPS technical complications and how to avoid them. AJR Am J Roentgenol 2011;196(03):675-685

89 Gaba RC. Transjugular intrahepatic portosystemic shunt creation with embolization or obliteration for variceal bleeding. Tech Vasc Interv Radiol 2016;19(01):21-35

90 Gupta AC, Wang W, Shah C, et al. Added value of covered stents in transjugular intrahepatic portosystemic shunt: a large singlecenter experience. Cardiovasc Intervent Radiol 2017;40(11): 1723-1731

91 Jung HS, Kalva SP, Greenfield AJ, et al. TIPS: comparison of shunt patency and clinical outcomes between bare stents and expanded polytetrafluoroethylene stent-grafts. J Vasc Interv Radiol 2009;20(02):180-185

92 Charon J-PM, Alaeddin FH, Pimpalwar SA, et al. Results of a retrospective multicenter trial of the Viatorr expanded polytetrafluoroethylene-covered stent-graft for transjugular intrahepatic portosystemic shunt creation. J Vasc Interv Radiol 2004;15 (11):1219-1230

93 Bucsics T, Schoder M, Diermayr M, et al. Transjugular intrahepatic portosystemic shunts (TIPS) for the prevention of variceal re-bleeding - a two decades experience. PLoS One 2018;13 (01):e0189414

94 Bucsics T, Schoder M, Goeschl N, et al. Re-bleeding rates and survival after early transjugular intrahepatic portosystemic shunt (TIPS) in clinical practice. Dig Liver Dis 2017;49(12): 1360-1367

95 Gaba RC, Couture PM, Lakhoo J. Gastroesophageal variceal filling and drainage pathways: an angiographic description of afferent and efferent venous anatomic patterns. J Clin Imaging Sci 2015;5 (01):61-66

96 Malloy PC, Grassi CJ, Kundu S, et al; Standards of Practice Committee with Cardiovascular and Interventional Radiological Society of Europe (CIRSE) Endorsement. Consensus guidelines for periprocedural management of coagulation status and hemostasis risk in percutaneous image-guided interventions. J Vasc Interv Radiol 2009;20(7, Suppl):S240-S249

97 Lopera JE. Role of emergency transjugular intrahepatic portosystemic shunts. Semin Intervent Radiol 2005;22(04):253-265

98 Azoulay D, Castaing D, Majno P, et al. Salvage transjugular intrahepatic portosystemic shunt for uncontrolled variceal bleeding in patients with decompensated cirrhosis. J Hepatol 2001;35(05):590-597

99 Helton WS, Belshaw A, Althaus S, Park S, Coldwell D, Johansen K. Critical appraisal of the angiographic portacaval shunt (TIPS). Am J Surg 1993;165(05):566-571

100 Rössle M, Haag K, Ochs A, et al. The transjugular intrahepatic portosystemic stent-shunt procedure for variceal bleeding. $\mathrm{N}$ Engl J Med 1994;330(03):165-171

101 LaBerge JM, Ring EJ, Gordon RL, et al. Creation of transjugular intrahepatic portosystemic shunts with the Wallstent endoprosthesis: results in 100 patients. Radiology 1993;187(02):413-420

102 McCormick PA, Dick R, Panagou EB, et al. Emergency transjugular intrahepatic portasystemic stent shunting as salvage treatment 
for uncontrolled variceal bleeding. Br J Surg 1994;81(09): 1324-1327

103 Le Moine O, Devière J, Ghysels M, et al. Transjugular intrahepatic portosystemic stent shunt as a rescue treatment after sclerotherapy failure in variceal bleeding. Scand J Gastroenterol Suppl 1994;207:23-28

104 Jalan R, Elton RA, Redhead DN, Finlayson ND, Hayes PC. Analysis of prognostic variables in the prediction of mortality, shunt failure, variceal rebleeding and encephalopathy following the transjugular intrahepatic portosystemic stent-shunt for variceal haemorrhage. J Hepatol 1995;23(02):123-128

105 Sanyal AJ, Freedman AM, Luketic VA, et al. Transjugular intrahepatic portosystemic shunts for patients with active variceal hemorrhage unresponsive to sclerotherapy. Gastroenterology 1996;111(01):138-146

106 Tyburski JG, Noorily MJ, Wilson RF. Prognostic factors with the use of the transjugular intrahepatic portosystemic shunt for bleeding varices. Arch Surg 1997;132(06):626-630, discussion 630-632

107 Chau TN, Patch D, Chan YW, Nagral A, Dick R, Burroughs AK. "Salvage" transjugular intrahepatic portosystemic shunts: gastric fundal compared with esophageal variceal bleeding. Gastroenterology 1998;114(05):981-987

108 Patch D, Nikolopoulou V, McCormick A, et al. Factors related to early mortality after transjugular intrahepatic portosystemic shunt for failed endoscopic therapy in acute variceal bleeding. J Hepatol 1998;28(03):454-460

109 Gerbes AL, Gülberg V, Waggershauser T, Holl J, Reiser M. Transjugular intrahepatic portosystemic shunt (TIPS) for variceal bleeding in portal hypertension: comparison of emergency and elective interventions. Dig Dis Sci 1998;43(11):2463-2469

110 Saxon RR, Mendel-Hartvig J, Corless CL, et al. Bile duct injury as a major cause of stenosis and occlusion in transjugular intrahepatic portosystemic shunts: comparative histopathologic analysis in humans and swine. J Vasc Interv Radiol 1996;7(04):487-497

111 Loffroy R, Favelier S, Pottecher P, et al. Transjugular intrahepatic portosystemic shunt for acute variceal gastrointestinal bleeding: Indications, techniques and outcomes. Diagn Interv Imaging 2015;96(7-8):745-755

112 Perello MP, Mur JP, Vives MS, et al. Long-term follow-up of transjugular intrahepatic portosystemic shunt (TIPS) with stent-graft. Diagn Interv Radiol 2019;25(05):346-352

113 Gaba RC, Omene BO, Podczerwinski ES, et al. TIPS for treatment of variceal hemorrhage: clinical outcomes in 128 patients at a single institution over a 12-year period. J Vasc Interv Radiol 2012;23(02):227-235

114 Zhu Y, Wang X, Xi X, Li X, Luo X, Yang L. Emergency transjugular intrahepatic portosystemic shunt: an effective and safe treatment for uncontrolled variceal bleeding. J Gastrointest Surg 2019;23(11):2193-2200

115 Orloff MJ, Hye RJ, Wheeler HO, et al. Randomized trials of endoscopic therapy and transjugular intrahepatic portosystemic shunt versus portacaval shunt for emergency and elective treatment of bleeding gastric varices in cirrhosis. Surgery 2015;157 (06):1028-1045

116 Orloff MJ, Vaida F, Haynes KS, Hye RJ, Isenberg JI, Jinich-Brook H. Randomized controlled trial of emergency transjugular intrahepatic portosystemic shunt versus emergency portacaval shunt treatment of acute bleeding esophageal varices in cirrhosis. J Gastrointest Surg 2012;16(11):2094-2111

117 Rosemurgy AS, Frohman HA, Teta AF, Luberice K, Ross SB. Prosthetic H-graft portacaval shunts vs transjugular intrahepatic portasystemic stent shunts: 18-year follow-up of a randomized trial. J Am Coll Surg 2012;214(04):445-453, discussion 453-455

118 Henderson JM. Surgery versus transjugular intrahepatic portal systemic shunt in the treatment of severe variceal bleeding. Clin Liver Dis 2006;10(03):599-612, ix
119 Barange K, Péron J-M, Imani K, et al. Transjugular intrahepatic portosystemic shunt in the treatment of refractory bleeding from ruptured gastric varices. Hepatology 1999;30(05): $1139-1143$

120 Lo GH, Liang HL, Chen WC, et al. A prospective, randomized controlled trial of transjugular intrahepatic portosystemic shunt versus cyanoacrylate injection in the prevention of gastric variceal rebleeding. Endoscopy 2007;39(08):679-685

121 Lakhoo J, Bui JT, Lokken RP, Ray CE Jr, Gaba RC. Transjugular intrahepatic portosystemic shunt creation and variceal coil or plug embolization ineffectively attain gastric variceal decompression or occlusion: results of a 26-patient retrospective study. J Vasc Interv Radiol 2016;27(07):1001-1011

122 Garcia-Tsao G. Management of acute variceal hemorrhage as a model of individualized care for patients with cirrhosis. Clin Gastroenterol Hepatol 2018;16(01):24-26

123 Monescillo A, Martínez-Lagares F, Ruiz-del-Arbol L, et al. Influence of portal hypertension and its early decompression by TIPS placement on the outcome of variceal bleeding. Hepatology 2004;40(04):793-801

124 García-Pagán JC, Caca K, Bureau C, et al; Early TIPS (Transjugular Intrahepatic Portosystemic Shunt) Cooperative Study Group. Early use of TIPS in patients with cirrhosis and variceal bleeding. N Engl J Med 2010;362(25):2370-2379

125 Garcia-Pagán JC, Di Pascoli M, Caca K, et al. Use of early-TIPS for high-risk variceal bleeding: results of a post-RCT surveillance study. J Hepatol 2013;58(01):45-50

126 Rudler M, Cluzel P, Corvec TL, et al. Early-TIPSS placement prevents rebleeding in high-risk patients with variceal bleeding, without improving survival. Aliment Pharmacol Ther 2014;40 (09):1074-1080

127 Lv Y, Yang Z, Liu L, et al; AVB-TIPS Study Group. Early TIPS with covered stents versus standard treatment for acute variceal bleeding in patients with advanced cirrhosis: a randomised controlled trial. Lancet Gastroenterol Hepatol 2019;4(08): 587-598

128 Deltenre P, Trépo E, Rudler M, et al. Early transjugular intrahepatic portosystemic shunt in cirrhotic patients with acute variceal bleeding: a systematic review and meta-analysis of controlled trials. Eur J Gastroenterol Hepatol 2015;27(09):e1-e9

129 Halabi SA, Sawas T, Sadat B, et al. Early TIPS versus endoscopic therapy for secondary prophylaxis after management of acute esophageal variceal bleeding in cirrhotic patients: a meta-analysis of randomized controlled trials. J Gastroenterol Hepatol 2016;31(09):1519-1526

130 Njei B, McCarty TR, Laine L. Early transjugular intrahepatic portosystemic shunt in US patients hospitalized with acute esophageal variceal bleeding. J Gastroenterol Hepatol 2017;32 (04):852-858

131 Sahagun G, Benner KG, Saxon R, et al. Outcome of 100 patients after transjugular intrahepatic portosystemic shunt for variceal hemorrhage. Am J Gastroenterol 1997;92(09):1444-1452

132 Tesdal IK, Filser T, Weiss C, Holm E, Dueber C, Jaschke W. Transjugular intrahepatic portosystemic shunts: adjunctive embolotherapy of gastroesophageal collateral vessels in the prevention of variceal rebleeding. Radiology 2005;236(01): 360-367

133 Wei B, Chen S, Li X, Tang CW. [Prevention of variceal rebleeding by TIPS combined with embolization of gastric coronary veins: a clinical controlled study]. Zhonghua Gan Zang Bing Za Zhi 2011; 19(07):494-497

134 Chen S, Li X, Wei B, et al. Recurrent variceal bleeding and shunt patency: prospective randomized controlled trial of transjugular intrahepatic portosystemic shunt alone or combined with coronary vein embolization. Radiology 2013;268(03):900-906

135 Wu XJ, Cao JM, Han JM, Li JS. [Long-term results of TIPS, TIPS with CVO and combined TIPS and portal azygous disconnection for 
the treatment of portal hypertension]. Zhonghua Wai Ke Za Zhi 2009;47(06):446-449

136 Shi Y, Tian X, Hu J, et al. Efficacy of transjugular intrahepatic portosystemic shunt with adjunctive embolotherapy with cyanoacrylate for esophageal variceal bleeding. Dig Dis Sci 2014;59 (09):2325-2332

137 Xue H, Yuan J, Chao-Li Y, et al. Follow-up study of transjugular intrahepatic portosystemic shunt in the treatment of portal hypertension. Dig Dis Sci 2011;56(11):3350-3356

138 Xiao T, Chen L, Chen W, et al. Comparison of transjugular intrahepatic portosystemic shunt (TIPS) alone versus TIPS combined with embolotherapy in advanced cirrhosis: a retrospective study. J Clin Gastroenterol 2011;45(07):643-650

139 Qi X, Liu L, Bai M, et al. Transjugular intrahepatic portosystemic shunt in combination with or without variceal embolization for the prevention of variceal rebleeding: a meta-analysis. J Gastroenterol Hepatol 2014;29(04):688-696

140 Schultheiß M, Giesler M, Maruschke L, et al. Adjuvant transjugular variceal occlusion at creation of a transjugular intrahepatic portosystemic shunt (TIPS): efficacy and risks of bucrylate embolization. Cardiovasc Intervent Radiol 2019;42 (05):729-736 